\title{
Pacer Found in Back-Up Mode
}

National Cancer Institute

\section{Source}

National Cancer Institute. Pacer Found in Back-Up Mode. NCI Thesaurus. Code C62880.

A device with a pacing function found in back-up mode. This may be an appropriate failsafe action (e.g. end of battery life), or be caused by device-malfunction or due to operator error. 